\title{
A Method for Source-Microphone Range Estimation in Reverberant Environments, Using an Array of Microphones of Unknown Relative Position
}

\author{
Denis L. McCarthy and Frank Boland \\ Trinity College Dublin \\ Dublin2 \\ Ireland
}

E-mail: demccart@tcd.ie, fboland@tcd.ie

\begin{abstract}
Estimating the range between an acoustic source and a microphone is a central problem in microphone-array processing. Although many approaches have been proposed in the literature, these tend to require knowledge of the relative microphone positions. We propose a range estimation method, for use in reverberant environments and where the relative positioning of the elements of a microphone-array is unknown. We demonstrate the effectiveness of this method under real, reverberant conditions.
\end{abstract}

Keywords - Range Estimation, Microphone Arrays

\section{INTRODUCTION}

Range estimation, between an acoustic source and multiple microphones, is a central problem in the field of microphone-array processing. Such estimates could inform decisions regarding microphone selection, allowing us to select the microphone nearest the source or farthest from some likely interference. Indeed, range estimates are required to calculate optimal filter weights for nearfield beamforming. Range estimates could also have application in determining appropriate dereverberation strategies as well as automated camera steering/focusing. Given knowledge of the relative microphone positions, the source-microphone range may easily be obtained from estimates of relative source location - an end to which a variety of solutions have been proposed.

Much work has focused on the use of time-delay estimates (TDEs) between microphones. In the two-dimensional case, source location may be considered a practical application of Apollonius' Problem of tangent circles. The mathematical solution to this problem, as discovered by Viete, may be easily expanded to the three-dimensional case and given TDEs between a minimum of four microphones (three in the two-dimensional case), a source location may be found. In [1], TDEs are determined for pairs of microphones in a series of four-element, square microphone arrays. From these, a series of source bearing-lines are calcu- lated, with the final source location estimate being calculated as a weighted average of the closest intersections between bearing-line pairs. In [2] the authors estimate the source location via a least squares fitting of the time delay estimates.

Several methods locate the source by a polling of candidate locations. In [3], the steered response power (SRP) is determined for a series of discrete locations, in the near-field of an array, with the maximum returned power being assumed to correspond to the source location. In [4] the authors formulate a approximate maximumlikelihood location estimator. This ML estimator, which they show to be equivalent to a weighted cross-correlation criterion, is then used to select the source location from among the candidate locations.

In non-reverberant acoustic environments, or in situations where the effects of reverberation are negligible, relative range estimates may be obtained from a comparison of received signal power. In [5] the authors combine TDEs and relative signal power measurements to determine the proximity and direction of arrival of a source in the extreme near-field of a two-element array. In [6] the authors present a method for source localisation that utilizes received signal energy only. Whilst this technique is reported as returning consistently accurate source-bearing estimates, range estimation is seen, in the presence of reverberation, to be 
subject to a significant bias.

Whilst many source localization algorithms have been shown to be effective in the presence of reverberation, these typically require knowledge of the relative microphone positions. For many practical applications, such information is unavailable or unreliable. Indeed, the question of how to estimate the range between a sound source and a microphone, in the presence of reverberation and with the relative positions of the microphones unknown, remains largely unaddressed. We propose a solution to this problem. Our method combines relative power measurements with TDEs to obtain absolute source-microphone range estimates for microphones at unknown locations. In Section 2 we derive a generalized function for estimating the source-microphone range in adverse acoustic conditions. In Section 3 we address the specific case of source-microphone range estimation in the presence of reverberation and in Section 4 we describe a series of experiments designed to test our method under simulated and real, reverberant conditions. We conclude in Section 5.

\section{RANGe Finding}

In air, the power of the received direct-path component of sound is inversely proportional to the source-microphone range, $r$, squared.

$$
P_{d p}(r) \propto \frac{1}{r^{2}}
$$

From this follows

$$
P_{d p}(e r) \propto \frac{e^{-2}}{r^{2}}
$$

$P_{d p_{0}}$ is the direct-path power received by some microphone, $m_{0}$, at a source-microphone range, $r_{0}$. From this, the direct-path power received by any microphone may be given as a function of $r$ and $r_{0}$

$$
P_{d p}(r)=P_{d p_{0}}\left[\exp \left(-2 \ln \left(\frac{r}{r_{0}}\right)\right)\right]
$$

From any one of a variety of time-delay estimation techniques (see [7] and references therein), we may obtain estimates of the absolute difference in the distances to the source between microphones.

$$
r_{x}-r_{0}=c \tau_{x}
$$

where $c$ is the speed of sound in air and $\tau_{x}$ is the time-delay estimate between $m_{x}$ and $m_{0}$. In noiseless, anechoic environments the direct-path sound accounts for all acoustic energy received by the microphones and so, by substituting (4) into (3) and performing algebraic manipulation, we obtain a simple and well known estimator of $r_{0}$.

$$
r_{0}=\frac{c \tau_{x} \sqrt{\frac{P_{d p}\left(r_{x}\right)}{P_{d p_{0}}}}}{1-\sqrt{\frac{P_{d p}\left(r_{x}\right)}{P_{d p_{0}}}}}
$$

However, in non-ideal acoustic environments, the ratio of received powers between pairs of microphones is distorted by the presence of interfering noise and reverberation. Equation (6) describes the power received by a microphone (direct sound and interference), as a function of $r$, normalized relative to the power received at $m_{x}$.

$$
p_{x}\left(r_{y}\right)=\frac{1}{P_{T_{x}}}\left\{P_{d p_{x}}\left[\exp \left(-2 \ln \left(\frac{r_{y}}{r_{x}}\right)\right)\right]+P_{I y}\right\}
$$

where $P_{I_{y}}$ is the received power due to interfering reverberation and noise at $m_{y}$ and $P_{T_{x}}=$ $P_{d p_{x}}+P_{I_{x}}$. In the general case, $P_{I y}$ is unknown but the ratio $\frac{P_{I y}}{P_{I x}}=\beta_{x, y}$ is assumed known. We now derive an estimator of $r_{0}$ for non-ideal acoustic environments.

$$
g_{x}\left(r_{x}, r_{y}\right)=\beta_{x, y} p_{x}\left(r_{x}\right)-p_{x}\left(r_{y}\right)
$$

$$
g_{x}\left(r_{x}, r_{y}\right)=\frac{P_{d p_{x}}}{P_{T_{x}}}\left\{\beta_{x, y}-\exp \left(-2 \ln \left(\frac{r_{y}}{r_{x}}\right)\right)\right\}
$$

From this

$$
\frac{g_{x}\left(r_{x}, r_{y}\right)}{g_{x}\left(r_{x}, r_{z}\right)}=\left\{\frac{\beta_{x, y}-\exp \left(-2 \ln \left(\frac{r_{y}}{r_{x}}\right)\right)}{\beta_{x, z}-\exp \left(-2 \ln \left(\frac{r_{z}}{r_{x}}\right)\right)}\right\}
$$

Substituting in (4)

$$
\frac{g_{x}\left(r_{x}, r_{y}\right)}{g_{x}\left(r_{x}, r_{z}\right)}=\left\{\frac{\beta_{x, y}-\exp \left(-2 \ln \left(1+\frac{c \tau_{y}-c \tau_{x}}{r_{0}+c \tau_{x}}\right)\right)}{\beta_{x, z}-\exp \left(-2 \ln \left(1+\frac{c \tau z-c \tau_{x}}{r_{0}+c \tau_{x}}\right)\right)}\right\}
$$

From (10) we see that $\frac{g_{x}\left(r_{x}, r_{y}\right)}{g_{x}\left(r_{x}, r_{z}\right)}$ is, in fact, a function of the known values $\tau_{x}, \tau_{y}, \tau_{z}, \beta_{x, y}$ and $\beta_{x, z}$ and a single unknown, $r_{0}$. Solving (10), which we shall refer to as the Range-Finder algorithm, gives us an estimate of $r_{0}$. 


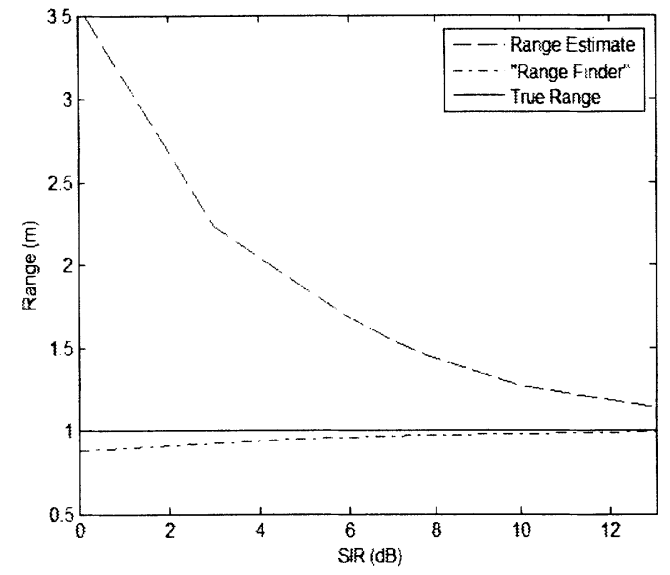

Fig. 1: A comparison of the performance of the Range-Finder and simple range estimator algorithms w.r.t SIR

To illustrate the performance of the RangeFinder under adverse acoustic conditions, a series of simple simulations was performed. Three microphones were simulated as being in an anechoic environment, at ranges $1 \mathrm{~m}, 2 \mathrm{~m}$ and $4 \mathrm{~m}$, respectively, from a source producing a "maximum-length sequence" (MLS) of 8191 samples in length. The microphone recordings were corrupted with uncorrelated white Gaussian interference signals. The performance of the Range-Finder algorithm was compared to that of the simple range estimator given in (5), with respect to the signal-to-interference ratio (SIR). The SIR taken is that at the microphone closest to the source and, in order to achieve the best possible performance, the simple range estimator uses the two microphones closest to the source only. For simplicity, $\beta_{x, y}=1, \forall x, y$. From the results, shown in Figure(1), it is clear that the simple range estimator suffers from a significant bias whilst the range-finder algorithm obtains accurate range estimates even at low SIR's.

\section{Range Finding in Reverberant Rooms}

Typically, in reverberant environments, both the received reverberant power, $P_{I}$, and the ratios of these powers between microphones, $\beta$, are unknown. We are forced, then, to use assumed values and so we take $\beta_{x, y}=1, \forall x, y$. In other words we assume that received reverberant energy levels are the same at each microphone. This assumption is a reasonable one in many cases, particularly when we are discussing long-term reverberation. However, short-term reverberation levels are heavily dependant on factors such as the proximity of a microphone to reflecting surfaces and the absorption/diffusion characteristics of these surfaces. We can expect greater levels of short-term reverberation in a microphone positioned next to a polished
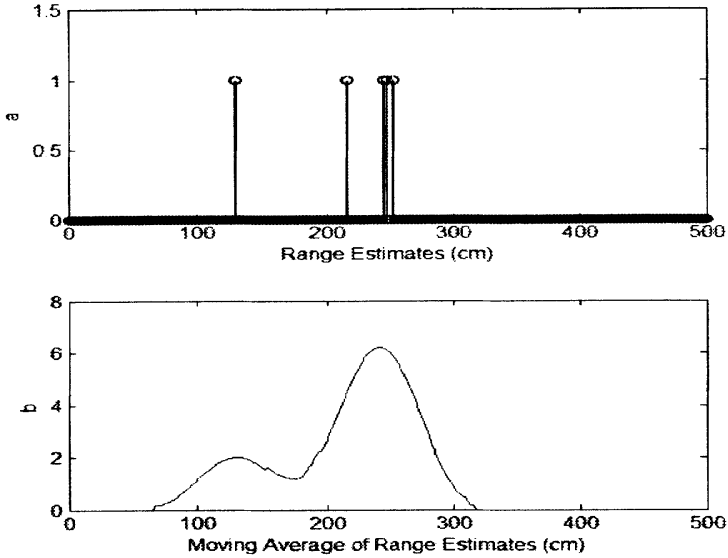

Fig. 2: The moving average, (b), is calculated by convloving the range-estimate pulse train, (a), with a Hamming window

table compared to one positioned next to, say, curtains. This may negatively impact the accuracy of our estimates. We note that reverberation may also effect the accuracy of the time-delay estimation algorithm used, however, a discussion of these effects and the means by which they may be mitigated against is beyond the scope of this paper and so is omitted here.

Considering this, we must take into account the possibility (indeed likelihood) that one or more microphones will receive levels of acoustic energy that do not correspond to the model assumed in (6). The inclusion of range estimates calculated using such microphones could lead to large errors. In practical situations, we would likely require more than three microphones, to ensure that we obtain at least one accurate range estimate. However, we are then presented with the problem of distinguishing between accurate and faulty results. We require an approach that may effectively discriminate between correct results and a comparable number of false estimates.

Possible solutions to the problem of erroneous estimates could involve averaging and/or the removal of outliers. Unfortunately, outliers may not be easily identifiable and the mean of the range estimates cannot always be assumed to approximate the true range, as the mean may be significantly affected by false results. Our first step therefore, is to identify and remove as many false results as possible.

Given any three microphones, there exists six possible permutations of $\left[m_{x}, m_{y}, m_{z}\right]$. All six of these yield identical estimates of $r_{0}$, therefore, using $M$ microphones, we may obtain up to ${ }^{M} \mathbf{C}_{3}$ independent range estimates. Some erroneous estimates may be easily spotted. Negative ranges, 


\begin{tabular}{|c|c|c|c|}
\hline & $x(m)$ & $y(m)$ & $z(m)$ \\
\hline$m_{0}$ & 1.5 & 3.5 & 1.75 \\
\hline$m_{1}$ & 2.5 & 2.5 & 1.75 \\
\hline$m_{2}$ & 1.5 & 1.5 & 1.8 \\
\hline$s$ & 1.5 & 4.81 & 1.07 \\
\hline
\end{tabular}

Table 1: Coordinates of the simulated microphones and source, $\mathrm{s}$

for example, may be ignored and so, by selecting $m_{0}$ such that it is the closest microphone to the source (this information can be obtained from the TDEs) we increase the likelihood that underestimates of the range are negative and, hence, easily identifiable as false. Indeed, given any known range boundary we can identify and remove erroneous estimates. Unfortunately, we are, very often, still left with several false but otherwise plausible range estimates.

The second step is to find a moving average of the remaining range estimates. Figure(2a) shows a pulse train corresponding to the estimates of a source-microphone range. Each sample in the pulse train corresponds to an increment in the range estimate, $\Delta r$. We obtain a moving average of these estimates by convolving them with a Hamming window, Figure(2b).

The moving average returns local maxima corresponding to clusters of estimates. The rational motivating this approach is that correct results will be grouped around the true range value while false results will demonstrate no such correlation.

\section{EXPERIMENTS}

A series of experiments were performed to test the performance of the Range-Finding algorithm. A room was simulated using an acoustic software package. The dimensions of the room were $[5.25 \mathrm{~m} 6.95 \mathrm{~m} 2.44 \mathrm{~m}]$. Three microphones and a sound source were placed at the locations given in Table(1). Using a raytracing algorithm, the first $20 \mathrm{~ms}$ of the source-microphone impulse responses were generated and statistical reverberant tails added. These impulse responses were then convolved with a MLS of length 32767 samples to obtain the simulated "recordings". The sampling rate used was $10 \mathrm{kHz}$. The reverberation time, $R T_{60}$, of the room was varied by altering the rooms surface absorption coefficients. The recordings were split into segments of 1024 samples and a range estimate for $m_{0}$ was found for each segment using both the Range-Finder algorithm and the simple range estimator given in (5). The mean of these results, plus and minus the standard deviation and with respect to $R T_{60}$, is shown in Figure(3).

To test the Range-Finder in real reverberant conditions, five microphones were setup at vary-

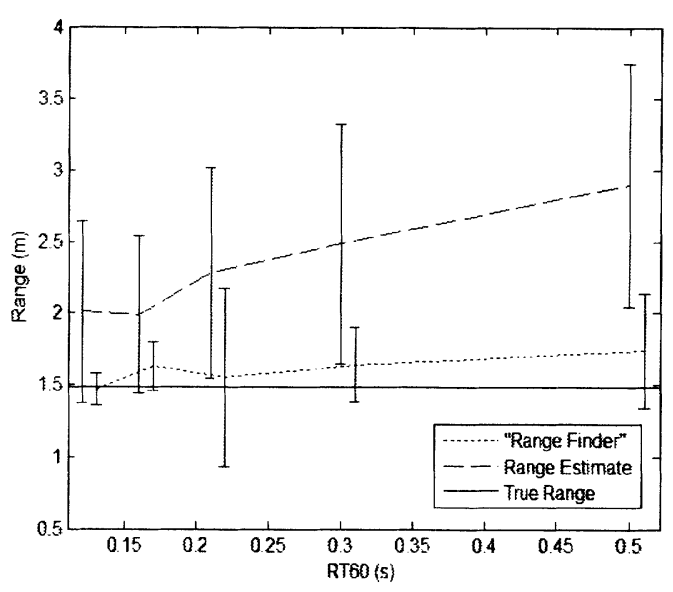

Fig. 3: A comparison of the performance of the Range-Finder and simple range estimator algorithms w.r.t $R T_{60}$

ing distances from a loudspeaker in a reverberant hall $\left(R T_{60}=1.1 s\right)$ and a medium-sized classroom $\left(R T_{60}=0.5 s\right)$. A maximum-length sequence (MLS) of approximately $5.6 \mathrm{~s}$ duration was produced by the loudspeaker and recorded using each of the microphones at a sampling rate of $10 \mathrm{kHz}$. The time delay between each of the recorded samples was determined using a phasealignment transform (PHAT). The speed of sound was taken as $340 \mathrm{~ms}^{-1}$. The samples were split into unwindowed segments and then processed by the range finding algorithm. The segment length used was 4096 samples. Once again range estimates were found using both the Range-Finder algorithm and the simple range estimator, for each segment.

In the case of the reverberant hall, an analysis of the initial range estimates revealed that estimates calculated using one of the microphones, Mic 4, showed significant error. Per segment, 6 of the 10 range estimates incorporated Mic 4 in their calculation. In the case of the classroom, it was seen that range estimates calculated using measurements from two microphones, Mic 1 and Mic 4 , again showed significant error. This meant that 9 of the 10 range estimates for each microphone were false.

From the initial range estimates, a moving average was found for both estimation methods. The range increment, $\Delta r$, was $1 \mathrm{~cm}$ and the Hamming window was 50 increments wide. The maximum value of this was taken to as $r_{0}$. The results are shown in Figure(4)

\section{Conclusion}

The problem of source-to-microphone range finding, in the presence of reverberation and without knowledge of the relative microphone locations is 


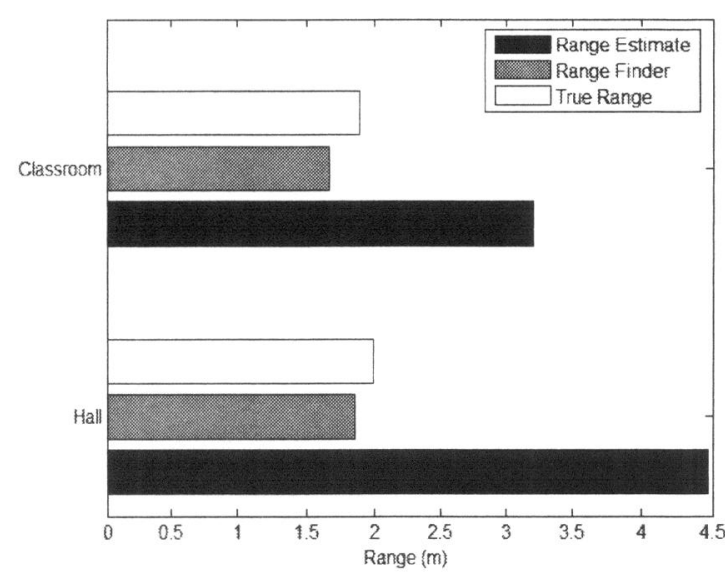

Fig. 4: Under real reverberant conditions, the Range-Finder outperforms the simple range estimator.

one that has remained largely unaddressed. In this paper we have proposed a solution to this problem that uses multiple microphones. Our method is robust against false results, even where the number of such results is comparable to, or, indeed, significantly greater than, the number of correct range estimates.

Future work will investigate, adapting our approach for cases where the acoustic source is a human voice. It is expected that such cases will present additional difficulty for the range finding algorithm. In real rooms, levels of received reverberant power are equal for all microphones in a broadband sense only. Large portions of human speech are spectrally sparse (mainly the harmonic or "voiced" sounds) and at individual, and in particular low frequencies, the frequency responses of differing source-microphone channels do not consistently conform to the model assumed herein. Preliminary results have shown range finding performance to be strongly effected by acoustic environment and microphone positioning and have indicated the need for more effective discrimination between accurate and spurious results.

\section{ACKNOWLEDGEMENTS}

The support of the Informatics Commercialisation initiative of Enterprise Ireland is gratefully acknowledged. Denis McCarthy also acknowledges the financial support, from Trinity College, of a postgraduate studentship.

\section{REFERENCES}

[1] Brandstein, M.S.; Adcock, J.E.; Silverman, H.F., "A closed-form method for finding source locations from microphone-array time-decay estimates," Acoustics, Speech, and Signal Processing, 1995. ICASSP-95., 1995 Interna- tional Conference on , vol.5, no.pp.3019-3022 vol.5, 9-12 May 1995

[2] Kung Yao; Hudson, R.E.; Reed, C.W.; Daching Chen; Lorenzelli, F., "Blind beamforming on a randomly distributed sensor array system," Selected Areas in Communications, IEEE Journal on , vol.16, no.8pp.1555-1567, Oct. 1998

[3] Griebel, S.M.; Brandstein, M.S., "Microphone array source localization using realizable delay vectors ," Applications of Signal Processing to Audio and Acoustics, 2001 IEEE Workshop on the , vol., no.pp.71-74, 2001

[4] Chen, J.C.; Hudson, R.E.; Kung Yao, "Maximum-likelihood source localization and unknown sensor location estimation for wideband signals in the near-field," Signal Processing, IEEE Transactions on [see also Acoustics, Speech, and Signal Processing, IEEE Transactions on], vol.50, no.8pp.1843-1854, Aug 2002

[5] Teutsch, H.; Elko, G.W., "An adaptive closetalking microphone array," Applications of Signal Processing to Audio and Acoustics, 2001 IEEE Workshop on the , vol., no.pp.163-166, 2001

[6] Birchfield, S.T.; Gangishetty, R., "Acoustic localization by interaural level difference," Acoustics, Speech, and Signal Processing, 2005. Proceedings. (ICASSP '05). IEEE International Conference on , vol.4, no.pp. iv/1109iv/1112 Vol. 4, 18-23 March 2005

[7] DiBiase, J.H.; Silverman, H.F.; Bransdstein, M.S., "Robust Localization in Reverberant Rooms", Microphone Arrays; Signal Processing Techniques and Applications, M. Branstein, D. Ward (Eds.), Springer Press 